\title{
Linfangiectasias en yeyuno como causa de sangrado digestivo oscuro manifiesto
}

\author{
Clinical case of lymphangiectasia in jejunum as a \\ cause of manifest obscure gastrointestinal bleeding
}

\author{
Albis Hani, Rómulo Vargas, Alejandro Concha, Reinaldo Rincón, \\ Gabriel Mosquera-Klinger, Valeria Costa, Ana Leguízamo, \\ Jhon Carvajal, Carolina Ardila • Bogotá, D.C. (Colombia)
}

\section{Resumen}

El sangrado digestivo oscuro constituye entre 10 y $20 \%$ de todas las causas de sangrado digestivo. La etiología está claramente relacionada con el grupo etario en que se presente. Las linfangiectasias del yeyuno son una causa rara de sangrado digestivo, pero puede llegar a ser de alta mortalidad en caso de que no se detecte tempranamente. Reportamos un caso de un paciente joven sin historia previa de enfermedad, con sangrado digestivo manifestado por melenas, con anemización severa, con estudios endoscópicos iniciales normales, en quien hacemos abordaje con estudios para intestino delgado (videocápsula endoscópica y enteroscopia anterógrada de doble balón), con los que hacemos el diagnóstico y es llevado a cirugía, con adecuada evolución clínica y curación de la enfermedad.

(Acta Med Colomb 2015; 40: 158-161).

Palabras claves: linfangiectasias, videocápsula endoscópica, enteroscopia anterógrada de doble balón, sangrado digestivo oscuro.

\begin{abstract}
Obscure gastrointestinal bleeding is between 10 to $20 \%$ of all causes of gastrointestinal bleeding. The etiology is clearly related to the age group in which it is presented. Lymphangiectasia in jejunum is a rare cause of gastrointestinal bleeding, but can have high mortality if not detected early. A case of a young patient with no history of previous disease presenting gastrointestinal bleeding manifested by melaena, with severe anemia and normal initial endoscopic studies, is presented. The diagnosis was made by endoscopic video capsule and antegrade double-balloon enteroscopy and surgery was performed with adequate clinical evolution and cure of the disease. (Acta Med Colomb 2015; 40: 158-161).
\end{abstract}

Keywords: lymphangiectasia, video capsule endoscopy, double-balloon enteroscopy anterograde, obscure gastrointestinal bleeding.
Dres. Albis Hani, Rómulo Vargas, Alejandro Concha, Reinaldo Rincón: Profesores de Gastroenterología y Endoscopia Digestiva, Pontificia Universidad Javeriana, Hospital Universitario San Ignacio; Dres. Gabriel Mosquera-Klinger, Valeria Costa, Ana Leguízamo y Jhon Carvajal: Gastroenterólogos, Pontificia Universidad Javeriana; Dra. Carolina Ardila: Medica, Pontificia Universidad Javeriana. Bogotá, D.C. (Colombia).

Correspondencia. Dr. Gabriel MosqueraKlinger. Bogotá, D.C. (Colombia).

E-mail: gami8203@yahoo.com

Recibido: 11/II/2014 Aceptado: 28/V/2015

\section{Introducción}

El sangrado digestivo es una patología muy frecuente y es la urgencia más común en gastroenterología; en los Estados Unidos es responsable de más de 300000 ingresos hospitalarios y más de 30000 muertes por año (1). A pesar de todas las estrategias diagnósticas y terapéuticas actuales tiene una mortalidad estimada entre 7 y $10 \%$ (2). La causa más frecuente es la enfermedad ulcerosa-péptica, seguida por el sangrado por várices esofágicas. Entre 10 y $20 \%$ de todos los pacientes con sangrado digestivo, no se les documenta lesiones causantes de sangrado en estudios iniciales. Esto se denomina sangrado digestivo oscuro y tiene dos formas de presentación: sangrado digestivo de origen oscuro manifiesto es en el que hay sangrado macroscópico documentado por el médico o el paciente, con estudios endoscópicos altos y bajos negativos; y el sangrado digestivo de origen oscuro oculto en el que hay sangre oculta en heces persistentemente positiva sin sangrado macroscópico y sin lograr evidenciar con los estudios endoscópicos iniciales el sitio de sangrado (3).

En el abordaje de los pacientes con sangrado digestivo oscuro tenemos que tener en cuenta la edad en que se presenta, ya que es un factor muy importante. En menores de 40 años las causas más frecuentes son: tumores de intestino delgado, como linfomas, carcinoides y adenocarcinomas; divertículo de Meckel, lesiones de Dieulafoy, síndrome de poliposis familiar y enfermedad de Crohn $(3,4)$. 
Las linfangiectasias intestinales son una causa rara de anemia por pérdidas gastrointestinales crónicas, y su presentación como sangrado de origen oscuro manifiesto es aún menos frecuente. Sin embargo, se han reportado casos en los que incluso ha habido sangrados masivos que amenazan la vida como es el caso de nuestro paciente. El sitio más frecuente donde se encuentran es el duodeno (5). El diagnóstico se realiza mediante la evaluación endoscópica y biopsia del sitio comprometido, o como alternativa con la pieza quirúrgica cuando se lleva a cabo la resección. En los casos en los que no se visualiza la lesión mediante endoscopia, ni colonoscopia, se puede detectar por videocápsula endoscópica o enteroscopia.

Presentamos el caso de un paciente joven sin antecedentes previos de enfermedad, con sangrado digestivo manifestado por melenas abundantes, con anemización severa, que requiere hospitalización para estudios.

\section{Descripción del caso}

Se trata de un paciente de 53 años, masculino, dedicado a expender carnes en supermercado de cadena de la ciudad de Bogotá, Colombia, quien consulta por astenia, adinamia, vértigo sin pérdida del tono postural, melenas y pérdida de peso de $3 \mathrm{Kg}$ aproximadamente de tres semanas de evolución.
Como antecedentes médicos presenta hipertrigliceridemia, en manejo con gemfibrozilo. No refería consumo de AINES, no había historia de exposición a tabaco o licor.

En el momento del ingreso se encuentra un paciente estable hemodinámicamente, con palidez mucocutánea, taquicárdico, sin otros hallazgos anormales. Se hace para ese momento una impresión diagnóstica de síndrome anémico y probable sangrado digestivo de origen alto. Se solicitan paraclínicos encontrando anemia microcítica, hipocrómica con hemoglobina de ingreso de $6.3 \mathrm{~g} / \mathrm{dL}$, y por anemia severa sintomática, se indica transfusión de glóbulos rojos empaquetados, siendo llamativo pobre rendimiento transfusional con hemoglobinas de control que oscilaron entre 7 y $8 \mathrm{~g} / \mathrm{dL}$.

Posteriormente se realiza estudio endoscópico de vías digestivas altas, sin encontrar signos de sangrado o lesiones y por este motivo se realiza colonoscopia total un día después evidenciando restos melénicos provenientes de la válvula ileocecal, hallazgo que indica como posible origen del sangrado foco en intestino delgado.

Se decide realizar posteriormente estudio de intestino delgado con videocápsula endoscópica (VCE), la cual evidencia lesión exofítica de aspecto tumoral en yeyuno proximal con sangrado activo (Figura 1) y se da a conocer el caso a grupo de cirugía general y en conjunto se decide

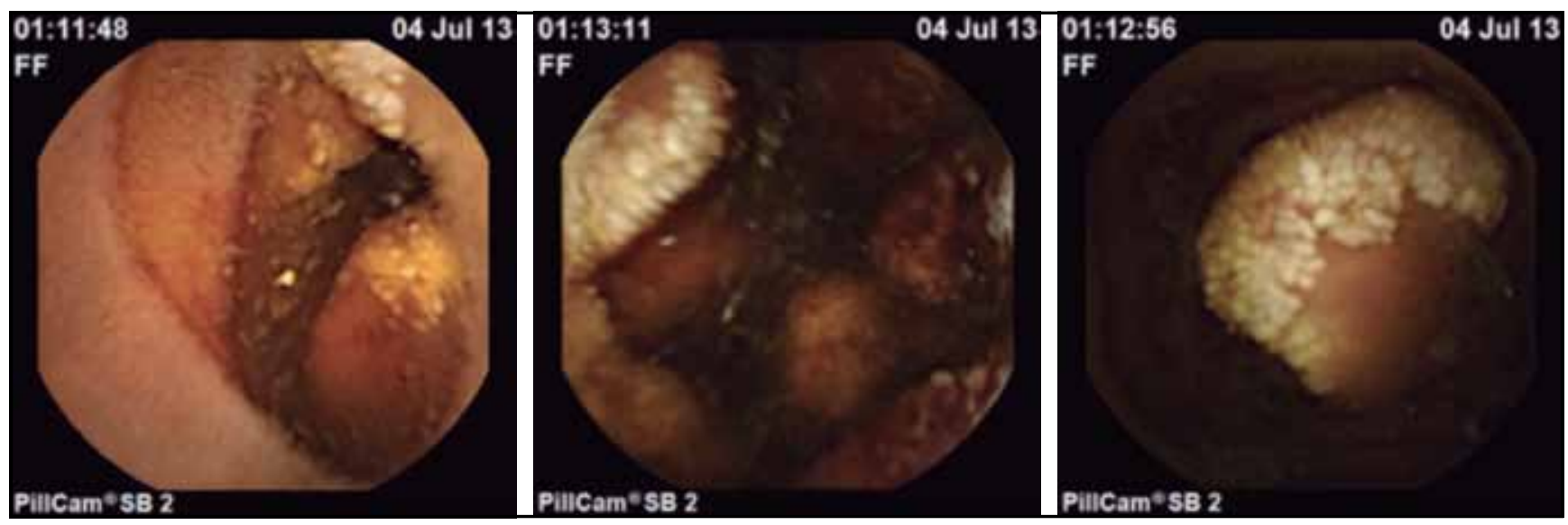

Figura 1. Imagen de la lesión en videocápsula endoscópica (Unidad de Gastroenterología HUSI).

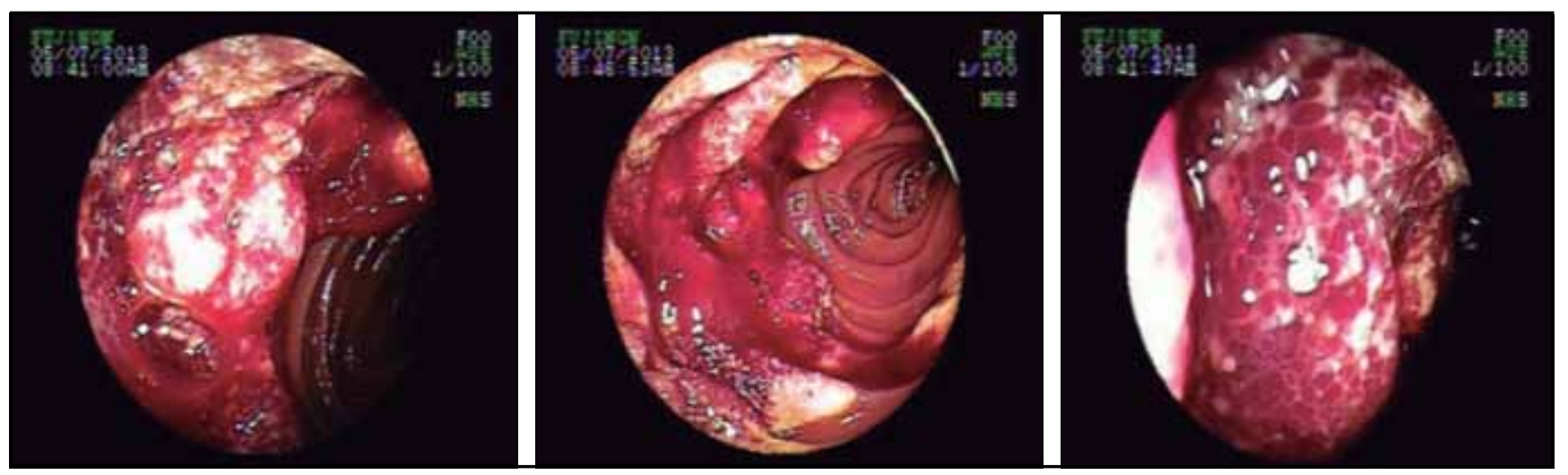

Figura 2. Aspecto de la lesión en enteroscopia de doble balón. Unidad de Gastroenterología HUSI. 
realizar tomografia de abdomen contrastada como parte de estudio de extensión y enteroscopia anterógrada de doble balón para valoración de lesión y marcación con tinta china para orientar resección quirúrgica.

Las características de la lesión documentada por enteroscopia de doble balón semejan una lesión de aspecto linfangiectásico y hemangiomatoso con sangrado en capa, que ocupaba $75 \%$ de la luz (Figura 2), en ese momento marcamos en la periferia de la lesión con tinta china diluida y la tomografía no documentó lesiones a distancia, con características imagenológicas que recuerdan lesión de origen linfangiectásico con engrosamiento de asas delgadas (Figura 3).

Posteriormente el paciente es llevado a resección quirúrgica por laparoscopia realizándose yeyunectomía proximal

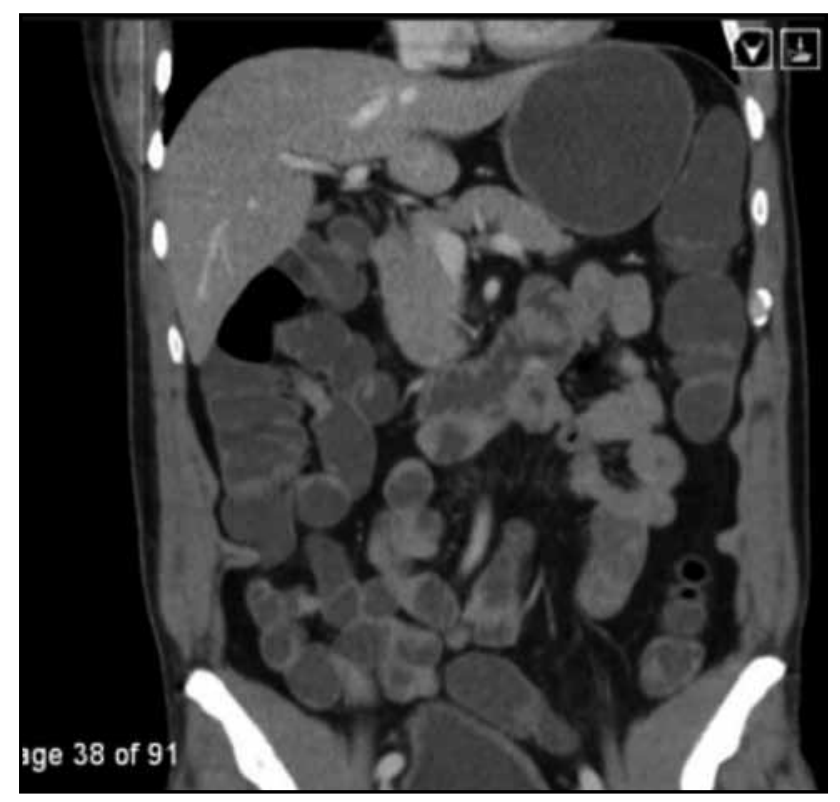

Figura 3. Engrosamiento de asas yeyunales en TAC. con hallazgo de masa marcada con tinta china a $100 \mathrm{~cm} \mathrm{del}$ ángulo de Treitz (Figura 4). La evolución posquirúrgica del paciente es adecuada, sin nuevos episodios de sangrado, con estabilización y aumento de niveles de hemoglobina. Se hace revisión del estudio de histopatológico de biopsias realizadas en enteroscopia y de la pieza quirúrgica, evidenciando hallazgos compatibles con linfangiectasias (Figura 5).

\section{Discusión}

Presentamos el caso de un paciente mayor de 40 años, sin historia previa de enfermedad. Con primer episodio de sangrado digestivo manifiesto, con clínica compatible con sangrado de origen en tracto digestivo alto, con anemización severa, que hace suponer un sitio de sangrado activo. Se hizo el abordaje diagnóstico inicial según el protocolo institucio-

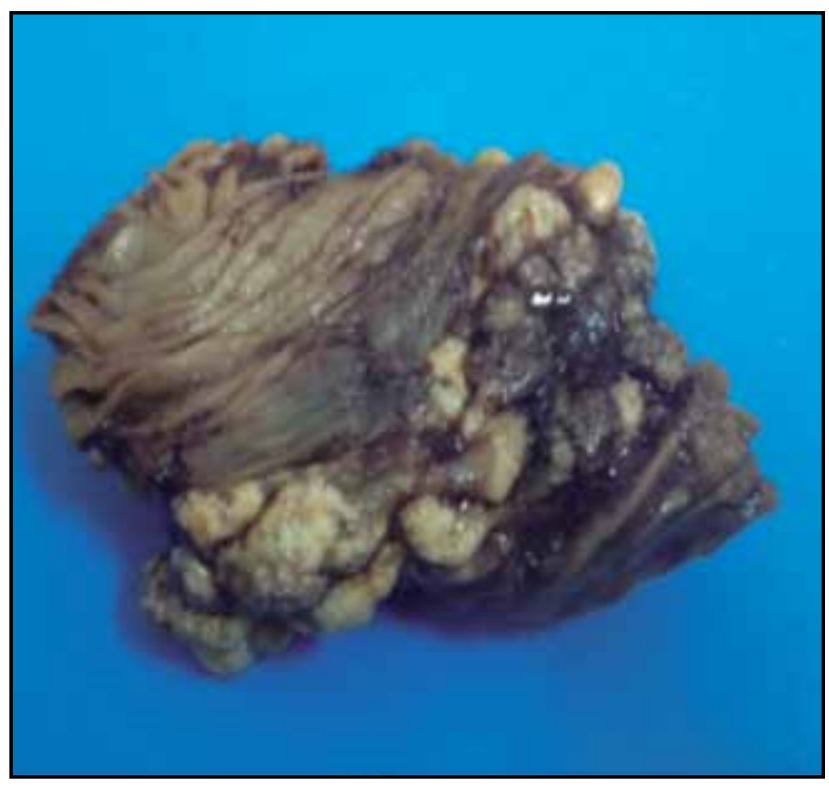

Figura 4. Pieza quirúrgica. Departamento Patología HUSI.

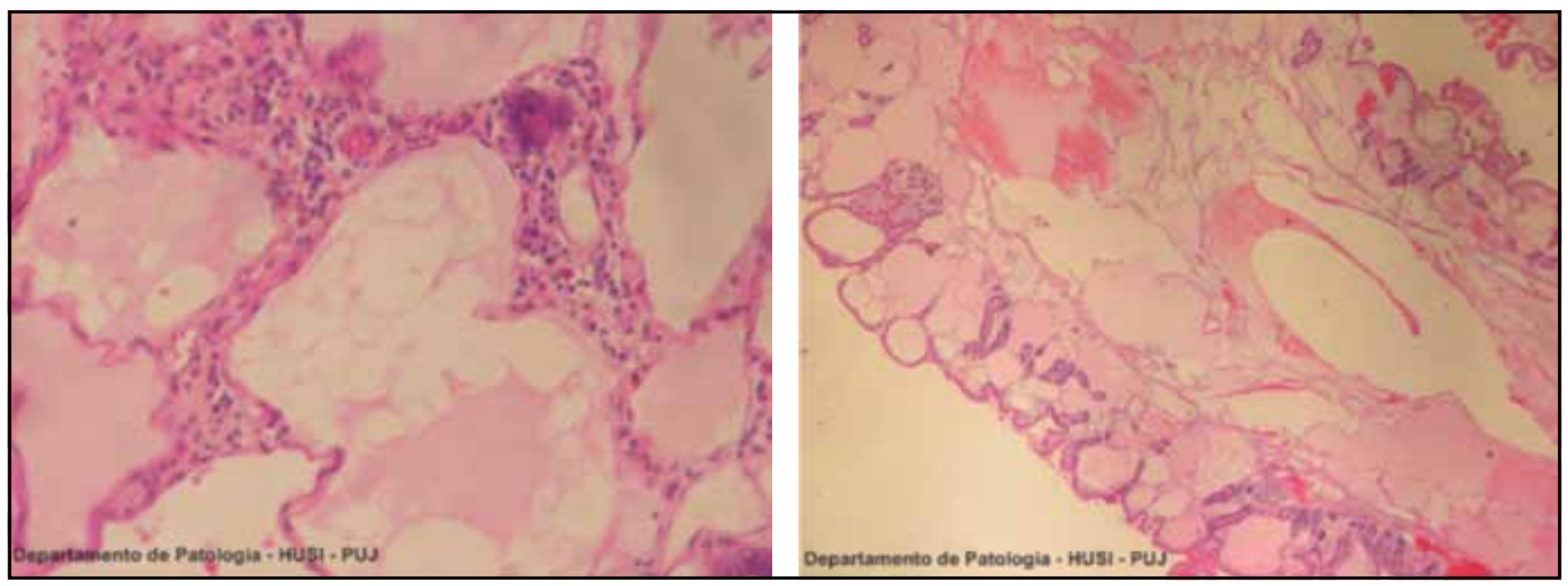

Figura 5. Imágenes de la biopsia de la lesión en hematoxilina eosina. 
nal con primera endoscopia digestiva alta, que no mostró sitio aparente de sangrado, y en colonoscopia evidencia de sangre proveniente de proximal. Hay autores que consideran hacer una nueva endoscopia alta teniendo en cuenta que pueden haber lesiones no evidenciables en una primera evaluación endoscópica hasta en 15-20\% de los casos. En este caso decidimos iniciar con VCE ya que es un método imagenológico no invasivo, fiable, que provee visualización completa y evaluación de la superficie y características de la mucosa del tracto digestivo superior e intestino delgado, y a su vez permite una adecuada ubicación de la lesión para determinar porque vía invasiva hacer diagnóstico histológico (enteroscopia anterógrada $v s$ enteroscopia retrograda) (6) o tratamiento quirúrgico definitivo. Los hallazgos descritos en VCE van desde lesiones blanquecinas y puntiformes que no tienen implicaciones patológicas, hasta la aparición de edema y engrosamiento de la pared llegando en ocasiones hasta lesiones polipoides con superficie blanquecina que refleja los linfáticos dilatados, de base amplia que pueden ser de gran tamaño causando incluso síntomas obstructivos $(7,8)$. Los hallazgos vistos en VCE en este caso nos hicieron tomar la acertada decisión de hacer enteroscopia anterógrada que nos sirvió para hacer diagnóstico histológico y además marcación para facilitar el tratamiento quirúrgico.

Las linfangiectasias intestinales pueden ser generalizadas o localizadas, dependiendo del sitio de obstrucción del drenaje linfático mesentérico (5). Se caracterizan clásicamente por la tríada de hipoproteinemia, edema y linfopenia. Cualquier proceso que obstruya el sistema linfático intestinal provocará la dilatación de los canales linfáticos, con malabsorción de quilomicrones, vitaminas liposolubles, y de proteínas (9), las manifestaciones clínicas son muy variadas que van desde síntomas generales, síntomas digestivos, síntomas de mal nutrición, pero en este caso nos parece llamativo que no tuvo ninguna manifestación clínica típica previamente, y su estado nutricional en general era bueno. En las linfangiectasias primarias, los síntomas se manifiestan temprano en la vida, al nacer o dentro de las primeras tres décadas. En general, cuanto más temprano sea la aparición de los síntomas, más grave es la enfermedad, caracterizada por edema masivo, diarrea y malabsorción. La muerte puede ocurrir temprano en tales casos (10).

Las linfangiectasias son causas raras de sangrado digestivo oscuro manifiesto en el grupo etáreo de nuestro paciente, y la localización en yeyuno aún hace más raro el caso, ya que la mayoría de veces se localizan en el duodeno (11).

Teniendo en cuenta que las linfangiectasias intestinales secundarias tienen múltiples diagnósticos diferenciales o pueden relacionarse con variadas afecciones como carcinomas abdominales o retroperitoneales, linfomas, fibrosis retroperitoneal, pancreatitis crónica, tuberculosis mesentérica, enfermedad de Crohn, enfermedad de Whipple, enfermedad autoinmune (tipo escleroderma y lupus eritematoso sistémico), pericarditis crónica, histiocitosis X y mieloma múltiple (12-14), se hizo al final de la hospitalización estudios adicionales para descartar de forma racional causas secundarias. Entonces en este caso consideramos que son linfangiectasias primarias.

\section{Conclusión}

Las linfangiectasias en yeyuno son una enfermedad poco común, por esta razón no hay evidencia suficiente en la literatura sobre cuál debe ser el seguimiento de los pacientes con presentación de linfangiectasias en intestino delgado. Con este paciente en particular nos queda el interrogante sobre el seguimiento: cuándo y con qué periodicidad debemos hacer estudios de control, ¿cuál es el método diagnóstico más apropiado para el seguimiento, estudios endoscópicos (VCE), enteroscopia o imágenes como la enteroTAC o enteroRMN? ¿O sólo hacer evaluación periódica de hemograma, sangre oculta en heces, o perfil nutricional?

\section{Referencias}

1. Fallah MA, Prakash C, Edmundowicz S. Acute gastrointestinal bleeding. Med Clin North Am 2000; 84(5): 1183-208.

2. Palmer K. Acute upper gastrointestinal hemorrhage. Br Med Bull 2007; 83: 307-24.

3. Lin S, Rockey D. Obscure Gastrointestinal Bleeding. Gastroenterol Clin N Am 2005; 34: 679-698.

4. ASGE. Obscure gastrointestinal bleeding. Gastrointest Endosc 2003; 58: 650.

5. Rashmi M. Niranjana Murthy B. Intestinal lymphangiectasia - a report of two cases. Indian J Surg 2010; 72: 149-151.

6. Pennazio et al. Small-bowel capsule endoscopy and device-assisted enteroscopy for diagnosis and treatment of small- bowel disorders: European Society of Gastrointestinal Endoscopy (ESGE) Clinical Guideline. Endoscopy 2015; 47: 352-376.

7. Kim JH, Bak YT, Kim JS, et al. Clinical significance of duodenal lymphangiectasia incidentally found during routine upper gastrointestinal endoscopy. Endoscopy 2009; 41: 510-515.

8. Camilleri M, Satti MB, Wood CB. Cystic lymphangioma of the colon. Endoscopic and histologicfeatures. Dis Colon Rectum 1982; 25: 813-816.

9. MamathaBhat, at al. Secondary intestinal lymphangiectasia due to multiple mieloma. Gastrointestinal Endoscopy 2011; 74: 718-720.

10. Warren Strober, Ivan J. Fuss. Protein-Losingenteropathies. Chapter 73. 12871309.

11. Freeman HJ and Nimmo, M. Intestinal lymphangiectasia in adults. World $J$ Gastrointest Oncology 2011; 3(2): 19-23.

12. RakeshRao, and HarohalliShashidhar. Intestinal lymphangiectasia presenting as abdominal mass. Gastrointestinal Endoscopy 2007; 65: No. 3.

13. BouhnikY et al. Very late onset small intestinal B cell lymphoma associated with primary intestinal 718 Gastrointestinal Endoscopy 2011; Volume 74, No. 3.

14. Hui C K. Intestinal lymphangiectasia associated with recurrence of histiocytosis X. Singapore Med J 2011; 52(9): 177-179. 